\title{
Quantity versus quality: Optimal methods for cell-free DNA isolation from plasma of pregnant women
}

Carolina J. Jorgez, $P h D^{1}$, Dianne D. Dang, BS ${ }^{1}$, Joe Leigh Simpson, $M D^{1,2}$, Dorothy E. Lewis, PhD ${ }^{3}$, and Farideh Z. Bischoff, $P h D^{1,2}$

\begin{abstract}
Purpose: Methods to isolate cell-free fetal DNA from maternal plasma are critical in developing noninvasive fetal DNA testing strategies. Given that plasma consists of heterogeneous DNA-size fragments in a complex mix of proteins, recovery and analysis of this DNA are understandably inefficient. To facilitate recovery, we performed qualitative and quantitative analysis of DNA isolated from maternal plasma. Methods: DNA isolated from maternal blood ( $n=15$ ) was compared using five different DNA isolation protocols: two conventional, two column-based, and one magnetic-bead based. Purity and concentration of DNA recovered were determined with a NanoDrop spectrophotometer. Real-time polymerase chain reaction quantification of the $\beta$-globin and DYS1 loci was performed to determine total and fetal-specific genome equivalents, respectively. Results: DNA quality and quantity were different among the five methods tested. Although purity and concentration of total DNA were greatest with the conventional boiling-lysis approach, correct detection of a male fetus was achieved in only $62.5 \%$ of cases. DNA isolation using the magnetic beads yielded the highest quantity of total DNA (2018.83 $\pm 4.09 \mathrm{GEq} / \mathrm{mL})$, with $100 \%$ fetal DNA detection. Conclusions: Optimal plasma DNA recovery protocols must take into account DNA purity and concentration. We confirm that the magnetic-beads method provides a fast, simple, sensitive, and specific approach to purify plasma DNA. The resulting high-quality DNA facilitates efficient examination of fetal DNA sequences. Genet Med 2006:8(10):615-619.
\end{abstract}

Detection and quantification of circulating fetal and total nucleic acids in maternal plasma has recently emerged as an alternative method for prenatal genetic diagnosis. Cell-free fetal DNA exists in plasma and serum of pregnant women as early as the sixth week of gestation, with concentrations increasing during pregnancy and peaking before parturition. ${ }^{1}$ Many laboratories, including ours, have shown the utility of fetal circulating DNA as a unique source of genetic material for noninvasive prenatal evaluation of fetal gender, genetic diseases, and aneuploidy. ${ }^{2-6}$ In addition, quantitative measurements of plasma DNA have correlated maternal risk for various pregnancy-related complications. ${ }^{7}$ Limitations in using cell-free fetal DNA in plasma for noninvasive prenatal analysis include concomitant recovery of abundant amounts of maternal DNA and failure to recover highly purified total DNA, both of which interfere with quantification strategies and assay sensitivity.

\footnotetext{
From the Departments of ${ }^{1}$ Obstetrics and Gynecology, ${ }^{2}$ Molecular and Human Genetics, and ${ }^{3}$ Immunology, Baylor College of Medicine, Houston, Texas.

Farideh Z. Bischoff, PhD, Department Of Obstetrics and Gynecology, Baylor College of Medicine, 2450 Holcombe Blvd.; RM M280, Houston, TX 77054.

Submitted for publication April 25, 2006.

Accepted for publication August 7, 2006.
}

DOI: 10.1097/01.gim.0000241904.32039.6f
Over the last few years, various protocols for plasma recovery and subsequent DNA isolation have been proposed. To separate plasma from whole blood, parameters including multiple centrifugations at different speed, filtration to remove contaminating intact or apoptotic cells, and removal of protein impurities have been explored. Only recently have reports attempted to compare DNA plasma isolation protocols. ${ }^{8-10}$ These reports are brief, often comparing only two approaches (e.g., manual vs. automated commercial methods) on the basis of outcome measures of recovered sequence specific copy numbers. However, variability of recovered DNA quantity and fetal sequence detection remains problematic, suggesting that additional measurements of DNA purity and concentration are necessary to evaluate plasma DNA isolation protocols and their utility for prenatal genetic testing. We have thus performed comparative analysis of two conventional and three commercial methods, by assessing measures of purity due to protein contamination, concentration of total DNA, and numbers of fetal specific sequences.

\section{MATERIALS AND METHODS}

\section{Sample collection and processing}

Under institutional review board approval from Baylor College of Medicine, 15 pregnant women (10-29 weeks gestation) gave informed consent to participate in the study. From each 
Table 1

Characteristics of different DNA isolation methods

\begin{tabular}{llccccc}
\hline & Magnetic-beads $^{a}$ & Qiamp-column $^{a}$ & Genelute-column $^{a}$ & Salting-out $^{b}$ & Boiling lysis $^{b}$ & ${\text { Albumin/IgG }+ \text { boiling lysis }^{c}}^{\text {Matrix }^{d}}$ \\
Magnetic-beads & Column & Column & NA & NA & NA \\
Proteinase K & Yes & Yes & Yes & Yes & No & No \\
Chaotropic salts & Yes & Yes & Yes & No & Yes & Yes \\
Harmful reagents & No & No & No & Yes & Yes & Yes \\
Time & $30 \mathrm{~min}$ & $30 \mathrm{~min}$ & $30 \mathrm{~min}$ & $60 \mathrm{~h}$ & $24 \mathrm{~h}$ & $26 \mathrm{~h}$ \\
\hline
\end{tabular}

NA, not applicable; Ig, immunoglobulin.

${ }^{a}$ Commercial methods.

${ }^{b}$ Conventional methods.

${ }^{c}$ Combination of commercial (deproteinization) and conventional methods.

${ }^{d}$ Matrix: component to which DNA binds.

patient, $20 \mathrm{~mL}$ of peripheral blood was collected in Vacutainers containing $1.5 \mathrm{~mL}$ of ACD Solution A (trisodium citrate, $22.0 \mathrm{~g} / \mathrm{L}$; citric acid, $8.0 \mathrm{~g} / \mathrm{L}$; and dextrose $24.5 \mathrm{~g} / \mathrm{L}$ ) and processed within 24 to 48 hours. Plasma was first separated from whole blood by centrifugation at $800 \mathrm{~g}$ for 10 minutes. Recovered plasma was then centrifuged for an additional 10 minutes at $13,500 \mathrm{~g}$ to remove residual intact cells. Finally, supernatant (containing free-DNA) was removed and stored at $-80^{\circ} \mathrm{C}$ up to 1 month in $800-\mu \mathrm{L}$ aliquots before DNA extraction.

\section{DNA extraction}

To extract DNA, $800 \mu \mathrm{L}$ of plasma from each maternal sample was used for each method. Table 1 shows a comparison of methods 1 to 5. Three commercial methods were compared. Extractions using commercial methods were performed according to the manufacturer's instructions with minor modifications to accommodate the $800-\mu \mathrm{L}$ volume of sample. The first method was based on the ability of DNA to absorb to the silica surface of magnetic particles in the presence of a chaotropic salt after proteinase $\mathrm{K}$ digestion using the DNA isolation kit from blood/bone marrow/tissue (method 1; Roche Diagnostics $\mathrm{GmbH}$, Mannheim, Germany), to be referred to as the magnetic-beads kit. The other two methods involved DNA extraction using a spin-binding mini-column in a chaotropic salt solution after proteinase K lysis using the Qiamp DNA blood mini kit (method 2; Qiagen Sciences, Germantown, MD), to be referred as the Qiamp-column kit, and the Genelute mammalian genomic DNA miniprep kit (method 3; Sigma-Aldrich, San Luis, MO), to be referred as the Genelute-column kit.

Extractions using two general conventional methods (4 and 5 ) were performed. The fourth method to be referred as salting-out involved dehydration and precipitation of proteins with a saturated $\mathrm{NaCl}$ solution and proteinase $\mathrm{K}$ digestion followed by phenol:chloroform:isoamyl alcohol extraction and ethanol precipitation of plasma DNA. This method followed the specifications of Miller et al., ${ }^{11}$ with minor modifications to accommodate the $800-\mu \mathrm{L}$ sample size. The fifth method to be referred as boiling lysis involved lysis of proteins by boiling in a chaotropic salt-phenol reagent followed by DNA extraction with chloroform:isoamyl alcohol and isopropanol precipitation. This method followed the specifications of Sandhu et al., ${ }^{12}$ with minor modifications to accommodate the $800-\mu \mathrm{L}$ sample size. To determine whether DNA concentration and purity are compromised by protein contamination, a sixth method, a variant of the boiling lysis method, involved the addition of an albumin/immunoglobulin (Ig)G column for deproteinization previous to DNA extraction using the Proteo-extract albumin/IgG removal kit maxi from Calbiochem (La Jolla, CA). In all methods, elution of DNA was performed using $100 \mu \mathrm{L}$ of elution buffer. All DNA samples were stored at $4^{\circ} \mathrm{C}$ before analysis.

\section{Measure of DNA concentration and purity}

To measure concentration and purity of recovered plasma, a NanoDrop ND-1000 spectrophotometer was used (NanoDrop Technologies, Wilmington, DE). This spectrophotometer required only $1 \mu \mathrm{L}$ of undiluted sample for assessment of concentration from $2 \mathrm{ng} / \mu \mathrm{L}$ to $3700 \mathrm{ng} / \mu \mathrm{L}$ of dsDNA. To measure DNA, the instrument automatically detects the high concentration and uses the $0.2-\mathrm{mm}$ pathlength to calculate the absorbance. Elution buffers used in each protocol were used as controls for optical density (OD) measures. Purity of the extracted DNA was based on the 260/280 O.D. ratio as calculated and displayed by the NanoDrop spectrophotometer.

\section{Polymerase chain reaction analysis}

For both the $\beta$-globin (102 base pair) and DYS1 (Y chromosome-specific sequence Y49a, 85 base pair) loci, quantitative real-time polymerase chain reaction (PCR) was performed using the Applied Biosystems 7700 sequence detection system (Foster City, CA). Quantitation of total and fetal DNA as genome equivalents per milliliter of plasma was based on copies of $\beta$-globin and DYS1 sequences detected per microliter of plasma. Each $50-\mu \mathrm{L}$ reaction contained $25 \mu \mathrm{L}$ of PE-ABI Universal Mastermix (Applied Biosystems) and $5 \mu \mathrm{L}$ of extracted DNA. Primers and probes were used at final concentrations of 300 and $200 \mathrm{~nm}$, respectively. Each reaction plate was run simultaneously with a duplicate calibration curve of titrated DNA (standard curve). Each sample was run in triplicate for both loci, and the mean of the values was determined using the 7700 software and the standard curve of known DNA concentrations. Cycling conditions for all reactions consisted sequen- 
tially of 2 minutes incubation at $50^{\circ} \mathrm{C}$ to activate UNGErase, initial denaturation step of $95^{\circ} \mathrm{C}$ for 10 minutes, and finally 40 cycles of $95^{\circ} \mathrm{C}$ for 15 seconds and $60^{\circ} \mathrm{C}$ for 60 seconds. All samples were analyzed blindly with respect to fetal gender. The standard factor of $6.6 \mathrm{pg}$ was used to convert the data to genome equivalents.

Primer and probe sequences were as follows:

DYS1 forward primer: $5^{\prime}$-TCC TGC TTA TCC AAA TTCACC AT-3'

DYS1 reverse primer: $5^{\prime}$-ACT TCC CTC TGA CAT TACCTG ATA ATT G-3'

DYS1 TaqMan probe: 5' -AAG TCG CCA CTG GAT ATCAGT TCC CTT GT-3'

$\beta$-globin forward primer: $5^{\prime}$-GTG CAC CTG ACT CCTGAG GAG A-3'

$\beta$-globin reverse primer: $5^{\prime}$-CCT TGA TAC CAA CCTGCC CAG-3'

$\beta$-globin TaqMan probe: $5^{\prime}$-AAG GTG AAC GTG GATGAA GTT GGT GG-3'

\section{RESULTS}

The mean and ranges of DNA concentration recovered in the five DNA extraction protocols using the spectrophotometer are shown in Table 2. Among the three commercial methods, the magnetic-beads kit yielded the highest concentration $(P>.001)$. DNA concentration using the magnetic-beads kit was 8.4- and 1.9-fold higher compared with the Qiamp-column and Genelute-column kits, respectively. Between the two conventional methods, the boiling lysis method yielded a 2.3fold increase in concentration compared with the salting-out method $(P=.147)$. However, intermethod comparisons reveal recovery using the boiling lysis method to be only 1.3 times higher than the magnetic-beads kit $(P=.436)$.
An albumin-IgG deproteinization column was used before the boiling lysis extraction (the method with the highest DNA concentration and purity by spectrophotometric determination) to determine whether DNA concentration and purity were compromised by protein contamination. This procedure increased DNA yield by 8.2 -fold, demonstrating that these two proteins may hinder high-yield DNA extraction. However, this procedure also reduced copy numbers for both $\beta$-globin and DYS1 sequences (Table 3), suggesting that DNA of interest (i.e., fetal) is bound to a protein complex that is subject to removal.

The purity of DNA extracted was determined on the basis of the $260 / 280$ OD ratio using a spectrophotometer. Pure DNA sample is expected to show a 260/280 ratio of 1.7/2.0. Table 2 summarizes the 260/280 ratio for the different methodologies. None yielded optimal ratios. The best ratio was achieved using the boiling lysis method (ratio 260/280 = $1.28 \pm 0.03$; Table 2); however, fetal DNA detection was correct in only five of eight cases $(62.5 \%)$ (Table 3$)$. When combined with the albuminIgG deproteinization column, the boiling lysis method yielded a significant increase in purity with a $260 / 280$ ratio $=1.55 \pm$ $0.04(P>.001)$ and correct fetal male detection in each of the four cases tested. In contrast, DNA purity was lower using the commercial magnetic-beads kit $(260 / 280$ ratio $=1.00 \pm 0.02$; Table 2), yet all eight fetal male cases were correctly identified.

Total cell-free DNA (maternal plus fetal) was also determined by real-time PCR for the ubiquitous $\beta$-globin gene. The mean and ranges of $\beta$-globin quantity with each of the isolation protocols are shown in Table 3. $\beta$-globin concentrations using the magnetic-beads kit were 1.7 -fold $(P=.408)$ and 8.9 -fold $(P>.01)$ higher compared with the Qiamp-column and Genelute-column kits, respectively. When quantities of $\beta$ globin between the two conventional methods were compared, no significant differences were observed $(P=.995)$. Contrary

Table 2

Comparison of the quality and quantity of DNA recovered from maternal plasma using different DNA isolation procedures as determined by spectrophotometer measurements

\begin{tabular}{|c|c|c|c|c|c|c|}
\hline & $\begin{array}{l}\text { Magnetic-beads }{ }^{a} \\
\mathrm{n}=15\end{array}$ & $\begin{array}{l}\text { Qiamp-column }^{a} \\
\mathrm{n}=15\end{array}$ & $\begin{array}{c}\text { Genelute-column }^{a} \\
\mathrm{n}=15\end{array}$ & $\begin{array}{l}\text { Salting-out } \\
\quad \mathrm{n}=15\end{array}$ & $\begin{array}{l}\text { Boiling lysis }^{b} \\
\mathrm{n}=15\end{array}$ & $\begin{array}{c}\text { Albumin/IgG }+ \text { boiling lysis }{ }^{c} \\
\mathrm{n}=11\end{array}$ \\
\hline $\begin{array}{l}\text { Range DNA } \\
\text { concentration }\end{array}$ & $13.10-52.9$ & $0.70-10.60$ & $6.70-85.60$ & $2.66-91.5$ & $9.65-297.4$ & $23.4-404.9$ \\
\hline $\begin{array}{l}\text { DNA concentration } \\
(\mathrm{ng} / \mu \mathrm{L})\end{array}$ & $35.39 \pm 0.22$ & $4.21 \pm 0.11^{d}$ & $18.61 \pm 0.29^{d}$ & $21.49 \pm 0.32$ & $48.89 \pm 0.56$ & $402.75 \pm 2.65^{e}$ \\
\hline $\begin{array}{l}\text { Range of 260/280 } \\
\text { ratio }\end{array}$ & $0.90-1.11$ & $0.18-2.85$ & $0.64-1.65$ & $0.54-1.48$ & $0.83-1.64$ & $1.24-2.00$ \\
\hline $260 / 280$ ratio & $1.00 \pm 0.02$ & $1.16 \pm 0.06$ & $0.81 \pm 0.04^{f}$ & $0.99 \pm 0.03^{e}$ & $1.28 \pm 0.03$ & $1.55 \pm 0.04^{e}$ \\
\hline $\begin{array}{l}\text { DNA concentration } \\
(\mathrm{ng} / \mu \mathrm{L})\end{array}$ & $35.39 \pm 0.22$ & $4.21 \pm 0.11^{d}$ & $18.61 \pm 0.29^{d}$ & $21.49 \pm 0.32$ & $48.89 \pm 0.56$ & $402.75 \pm 2.65^{e}$ \\
\hline
\end{tabular}

Ig, immunoglobulin.

${ }^{a}$ Commercial methods.

${ }^{b}$ Conventional methods.

${ }^{c}$ Combination of commercial (deproteinization) and conventional methods.

Values are expressed as average \pm standard error of the mean.

${ }^{d} P>.001 ;{ }^{e} P>.01 ;{ }^{f} P>.1$. 
Table 3

Comparison of the quantity of DNA recovered from maternal plasma using different DNA isolation procedures as determined by real-time polymerase chain reaction

\begin{tabular}{|c|c|c|c|c|c|c|}
\hline & $\begin{array}{l}\text { Magnetic-beads }{ }^{a} \\
\quad \mathrm{n}=15\end{array}$ & $\begin{array}{l}\text { Qiamp-column }{ }^{a} \\
\mathrm{n}=15\end{array}$ & $\begin{array}{c}\text { Genelute-column }{ }^{a} \\
\mathrm{n}=15\end{array}$ & $\begin{array}{l}\text { Salting-out }{ }^{b} \\
\mathrm{n}=15\end{array}$ & $\begin{array}{l}\text { Boiling lysis }^{b} \\
\mathrm{n}=15\end{array}$ & $\begin{array}{c}\text { Albumin/IgG + boiling lysis } \\
\text { n }=11\end{array}$ \\
\hline Range $\beta$-globin ${ }^{d}$ & $249.0-3054.8$ & $350.0-4346.3$ & $18.0-2505$ & $706.0-6195.3$ & $6.5-7151.8$ & $216-1419$ \\
\hline$\beta$-globin $(\mathrm{GEq} / \mathrm{mL})$ & $2018.83 \pm 4.09$ & $1172.58 \pm 2.12$ & $224.78 \pm 1.68^{g}$ & $1475.62 \pm 2.55$ & $1479.07 \pm 2.80$ & $553.50 \pm 1.99^{h}$ \\
\hline Range DYS $1^{e}$ & $52.0-220.0$ & $12.0-92.5$ & $15.0-43.75$ & $23.8-192.5$ & $14.8-324.5$ & $11.8-127.8$ \\
\hline DYS1 (GEq/mL) & $58.77 \pm 0.57$ & $23.55 \pm 0.38^{g}$ & $6.82 \pm 0.24^{f}$ & $60.00 \pm 0.55$ & $74.02 \pm 0.73$ & $37.59 \pm 0.62$ \\
\hline $\begin{array}{l}\text { Correct DYS1 } \\
\quad \text { detection (\%) }\end{array}$ & $8(100 \%)$ & $7(87.5 \%)$ & $4(50 \%)$ & $7(87.5 \%)$ & $5(62.5 \%)$ & $4(100 \%)$ \\
\hline $\begin{array}{l}\text { False DYS1 } \\
\quad \text { detection (\%) }\end{array}$ & 0 & 0 & 0 & $3(42.9 \%)$ & $1(14.3 \%)$ & $3(42.9 \%)$ \\
\hline
\end{tabular}

${ }^{a}$ Commercial methods.

${ }^{b}$ Conventional methods.

${ }^{c}$ Combination of commercial (deproteinization) and conventional methods.

d $\beta$-globin quantification is measure of total (maternal and fetal) DNA.

${ }^{e} D Y S 1$ quantification is a measure of only fetal DNA ( $Y$-chromosome sequences).

Values are expressed as average \pm standard error of the mean.

${ }^{f} P>.001 ;{ }^{g} P>.01 ;{ }^{h} P>.1$.

to spectrophotometer results, the intramethods comparison indicates that the highest quantities of DNA by $\beta$-globin determination was achieved using the magnetic-beads kit (1.3 times higher than the boiling lysis; $P=.619$ ), although this was not significant. The use of an albumin-IgG deproteinization column before performing boiling lysis extraction produced a decrease in $\beta$-globin copies by 2.7 -fold $(P=.100)$.

Finally, detection and quantity of cell-free fetal DNA were determined by measuring the number of DYS1 sequences (male DNA) for each isolation protocol (Table 3). Among the three commercial methods, the magnetic-beads kit yielded 2.5- and 8.6-fold higher levels of DYS1 than the Qiamp-column and Genelute-column kits, respectively. These concentrations are similar in variation to that based on $\beta$-globin determination. No false-negative DYS1 sequences were detected. When DYS1 concentrations among the conventional methods were compared, boiling lysis resulted in higher concentrations than the combination of either albumin-IgG deproteinization with boiling lysis (1.9 times) or salting-out (1.2 times). However, sensitivity was seven of eight $(87.5 \%)$ and five of eight (62.5\%) for male fetal DNA detection after the salting-out and boiling methods, respectively (Table 3). After comparison of DYS1 levels among all methods, the boiling lysis method was only 1.3 times higher than the magneticbeads kit, but not significantly different $(P=.678)$.

\section{DISCUSSION}

Two limiting factors must be taken into account when selecting protocols for isolation of plasma DNA for fetal DNA sequences: (1) the low quantity of fetal DNA available for isolation and (2) the existence of DNA in the form of complexes tightly bound with serum proteins. To help select a method that addresses these potentially contradictory factors, we determined the quality and quantity of DNA recovered using five different protocols for plasma DNA isolation. Concentration and purity as assessed by spectrophotometer, as well as the differences between copy number of both total $(\beta$-globin $)$ and fetal (DYS1) sequences, varied widely. We conclude, however, that detection of total and fetal sequences is best using magneticbeads, although the greatest yield in purity was achieved by the conventional boiling lysis method.

In comparison with the conventional method (Table 1), commercial kits provide a simple, fast, and safe way to separate aqueous and organic phases to purify DNA. None of the commercial kits gave false-positive results, suggesting high specificity of these methods for fetal cell-free DNA isolation. Although the three kits use the same principle, the magnetic-beads kit proved to be the most efficient in overall yield and purity of DNA with the smallest range of variation. In addition, the magnetic-beads kit detected all eight male cases without false-positive results. A possible explanation for the salutary results is that the magnetic-beads kit maximizes surface contact between the matrix and DNA, permitting better absorption of the DNA to the silica surface of the magnetic-beads. This result correlates with other studies using magnetic beads that indicate the ability of the beads to enhance DNA isolation is by amount and quality when compared with other commonly used techniques. ${ }^{13}$ In this study, the best results were obtained with the magnetic-beads kit; however, the Qiamp-column kit may also be reliable with an $87.5 \%$ detection of male fetuses. Both methods yielded satisfactory values of $\beta$-globin and DYS1 sequences with acceptable purity.

The two conventional methods used in this study were based on either salting-out of proteins ${ }^{11}$ or lysis of proteins by boiling. ${ }^{12}$ The boiling lysis method produced an overall higher DNA yield and quality (Table 2) than the salting-out method. The former showed only a small difference compared with the optimal commercial protocol of magnetic beads. However, the total DNA measured by real-time PCR using the $\beta$-globin loci in conventional methods was lower compared with the magnetic-beads kit (Table 3). It is possible that DNA concentration 
values estimated by spectrophotometer for the conventional methods were overestimated because of contaminants like proteins and/or salts (used to precipitate the DNA in these methods). Contaminants could also be responsible for lower sensitivity because of "carryover" of phenol or salts that could inhibit or reduce the efficiency of downstream applications such as PCR, and generate false-positives and/or negatives. Moreover, the conventional methods are time-consuming and have undesirable exposure to harmful chemicals (phenol and chloroform).

Plasma DNA extraction procedures rely on efficient separation of cell-free DNA from serum proteins. Proteinase K is commonly used as part of the deproteinization step incorporated in both commercial and conventional methods. As an alternative, a deproteinization method using commercial columns could be used for removal of more than $80 \%$ of plasma albumin and IgG, a crucial step for obtaining a high-quality DNA sample. Purity of the extracted DNA is traditionally confirmed by the spectrophotometer and calculated as the 260/280 OD ratio. A pure sample of DNA will have a relatively high 260/280 ratio (i.e., 1.7-2.0). We observed 260/280 OD ratios less than optimal in all of the samples; thus, DNA purity and concentrations as measured in this study are presumably influenced by the extent to which proteins are fully removed. We observed that as levels of protein removal from plasma increased, DNA purity and concentration improved but conversely reduced detection of total and fetal sequences. We proposed that the decrease in fetal sequence detection is the result of loss of smaller-size DNA fragments. As we showed by the use of an albumin-IgG deproteinization column before boiling lysis extraction, an increased yield and quality of DNA by as much as 8.2 -fold (Table 2) can be achieved. These results clearly demonstrate that removal of the most abundant proteins from plasma yields better-quality DNA. However, total and fetal DNA quantity measured by real-time PCR is concomitantly decreased by 2.6- and 1.6-fold, respectively (Table 3). Small fragments of cell-free (fetal and maternal) DNA are presumably lost with column-based deproteinization. Nevertheless, there is an increase in the percentage of recovered fetal sequences over total DNA (36\%), indicating enrichment for fetal sequences. If DNA isolation following column-based deproteinization can be improved, this approach could be potentially useful for further enrichment of fetal DNA.

This study showed a direct correlation between the purity of the samples and the amount of maternal and fetal sequences detected in both commercial and conventional DNA isolation methods. By using the column-based approach, we found that the two samples with the best 260/280 ratio also yielded the highest $\beta$-globin and DYS1 levels. With the use of the magnetic-beads method, the range of a 260/280 ratio was narrow (0.90-1.11). However, the two samples with the lowest $\beta$-globin concentration had a 260/ 280 ratio below 0.95 . The same trend was also observed with the conventional methods. Of the 15 samples extracted using the boiling-lysis method, 5 had a 260/280 ratio lower than 1.10, 4 of which had the lowest $\beta$-globin concentration (154.06 \pm 4.36 ) among the group. Also, DYS1 sequences were not detected in three of the male cases. With the salting-out method, the sample with the lowest 260/280 ratio also had the lowest $\beta$-globin concentration. All these data support a correlation between the purity and the detection efficiency of DNA sequences.

The purity of the sample is not just an indicator of total quantity of DNA recovered, but specifically of fetal DNA. Of the six methods, the average of 260/280 ratio of the four samples with the highest DYS1 levels was $1.26 \pm 0.03$. This value was higher than the average of all but the albumin method. In addition, among all methods of the 10 samples for which we were not able to detect DYS1 sequences, the average of 260/280 ratio was $0.70 \pm 0.05$, a value that was significantly different from samples with the highest $262 / 280$ ratio levels $(P=.0002)$. These data establish that the purity of the sample could be used as an indicator of the quality and quantity of DNA recovered from plasma.

In conclusion, five different methods for isolation of DNA from plasma were evaluated. A commercially available magnetic-beads kit provides optimal results for DNA purification with high sensitivity and specificity. The resulting high-quality DNA should facilitate precise quantification and sequence analysis, and enable more efficient examination into the molecular nature of the circulating DNA in maternal plasma.

\section{ACKNOWLEDGMENTS}

This research was supported in part by grant National Institutes of Health/National Institute of Child Health and Human Development HDO46623.

\section{References}

1. Lo YM, Tein MS, Lau TK, Haines CJ. Quantitative analysis of fetal DNA in maternal plasma and serum: implications for noninvasive prenatal diagnosis. Am J Hum Genet 1998;62:768-775.

2. Bischoff FZ, Lewis DE, Simpson JL. Cell-free fetal DNA in maternal blood: kinetics, source and structure. Hum Reprod Update 2005;11:59-67.

3. Johnson KL, Dukes KA, Vidaver J, LeShane ES. Interlaboratory comparison of fetal male DNA detection from common maternal plasma samples by real-time PCR. Clin Chem 2004;50:516-521.

4. Bischoff FZ, Nguyen DD, Marquez-Do D, Moise KJ. Noninvasive determination of fetal RhD status using fetal DNA in maternal serum and PCR. J Soc Gynecol Investig 1999;6:64-69.

5. Bischoff FZ, Dang DX, Marquez-Do D, Martinez D. Detecting fetal DNA from dried maternal blood spots: another step towards broad scale non-invasive prenatal genetic screening and feasible testing. Reprod Biomed Online 2003;6:349-351.

6. Bischoff FZ, Sinacori MK, Dang DD, Marquez-Do D. Cell-free fetal DNA and intact fetal cells in maternal blood circulation: implications for first and second trimester non-invasive prenatal diagnosis. Hum Reprod Update 2002;8:493-500.

7. Hahn S, Huppertz B, Holzgreve W. Fetal cells and cell free fetal nucleic acids in maternal blood: new tools to study abnormal placentation? Placenta 2005;26:515-526.

8. Huang DJ, Zimmermann BG, Holzgreve W, Hahn S. Use of an automated method improves the yield and quality of cell-free fetal DNA extracted from maternal plasma. Clin Chem 2005;51:2419-2420.

9. Chiu RW, Lui WB, El Sheikhah, A, Chan AT. Comparison of protocols for extracting circulating DNA and RNA from maternal plasma. Clin Chem 2005;51:2209-2210.

10. Tag CG, Schifflers MC, Mohnen M, Gressner AM. Improved method for isolating cell-free DNA. Clin Chem 2005;51:1561-1562.

11. Miller SA, Dykes DD, Polesky HF. A simple salting out procedure for extracting DNA from human nucleated cells. Nucleic Acids Res 1988;16:1215.

12. Sandhu GS, Kline BC, Stockman L, Roberts GD. Molecular probes for diagnosis of fungal infections. J Clin Microbiol 1995;33:2913-2919.

13. Stemmer C, Beau-Faller M, Pencreac'h E, Guerin E. Use of magnetic beads for plasma cell-free DNA extraction: toward automation of plasma DNA analysis for molecular diagnostics. Clin Chem 2003;49:1953-1955. 